\title{
Domestic injuries and suicide among women of reproductive age in Iran
}

This article was published in the following Dove Press journal:

International Journal of General Medicine

19 June 2012

Number of times this article has been viewed

\section{Zahra Fardiazar' \\ Homayoun Sadeghi- \\ Bazargani ${ }^{2}$ \\ Reza Mohammadi ${ }^{3}$}

'Women's Reproductive Health Research Center, ${ }^{2}$ Neuroscience Research Center, Tabriz University of Medical Sciences, Tabriz, Iran; ${ }^{3}$ Public Health Department, Karolinska Institute, Stockholm, Sweden
Correspondence: Homayoun SadeghiBazargani

Department of Public Health Sciences, World Health Organization Collaborating Center for Safe Communities, Karolinska Institute, Second Floor, Norrbacka, SE-17I76, Stockholm, Sweden

Tel +4686730212

Fax +4686730212

Email homayoun.sadeghi@gmail.com
Background: The aim of this study was to map out some epidemiological aspects of intentional and unintentional injuries among Iranian women of reproductive age using a national registry.

Methods: Injury data were taken from a national-based injury surveillance system over the period 2000-2002. The study population comprised $31.5 \%$ of the population of Iran.

Results: Of all the 307,064 domestic injuries reported during the years 2000-2002, about 152,600 cases $(49.7 \%)$ involved women. About half of these women $(76,474)$ were in the reproductive age group. The majority $(42.7 \%)$ of injuries among women of reproductive age were burn wounds followed by lacerations in $32.6 \%$. Eighty-five percent of suicide cases were poisonings, followed by $11 \%$ for suicides by burning. However, $45.2 \%$ of burn suicides were fatal, compared with a $0.89 \%$ fatality rate for poisonings. Of all female suicide victims, 1029 died, 174 victims became disabled, while the remainder improved or were undergoing therapy when reported.

Conclusion: Injuries, especially burns, are a major public health problem for women of reproductive age.

Keywords: injuries, women's health, accidents, burns, falls, poisonings, home safety, domestic injuries, epidemiology

\section{Introduction}

The traditional view of injuries as "accidents" or random events, has resulted in historical neglect of this important area of public health. ${ }^{1}$ Injury epidemiology can be defined as "the study of the distribution and determinants of injuries and safety-related states/events in specified populations, and the application of this study to prevent injuries and promote safety". ${ }^{2}$ Reports of injuries, if based on large-scale studies or widespread registries, are appropriate for focusing on specific populations prone to particular diseases and injuries.

The home, which is considered by many people to be a safe haven for living, may not prove to be so, according to statistics for domestic injuries worldwide. Children and women can be at higher risk of injuries in the home due to greater exposure to home hazards. Much research has been done on these injuries, especially during recent decades. ${ }^{3,4}$ However, most of the available knowledge is based on hospital data, and research data showing details of domestic injuries among women of reproductive age, especially from low-income and middle-income countries, are limited. The aim of this study was to map out some epidemiological aspects of intentional and unintentional injuries among Iranian women of reproductive age using a national registry. 


\section{Materials and methods}

Injury data were taken from a national-based injury surveillance system for the period 2000-2002. The system included registration forms for home-related injuries treated in health or emergency centers. In addition, a hierarchical reporting system was designed to gather data from all over the country. The surveillance system used in this study was based on the work of 31 Iranian universities in medical sciences and health services that are responsible for both medical education and delivering health services. All catchment areas were encompassed, but some universities initiated their program with just a portion of their population.

The study population comprised $31.5 \%$ of the population of Iran. The register was initially constructed in order to map the epidemiology of home-related injuries sustained in the country. In Iran, the population in rural areas, but not that in all urban areas, is well defined, which means that the incidence rate in this study is valid mostly for the former segment of the population and small cities.

The variables for injury recording included a minimum data set which was tested in a pilot program. ${ }^{5}$ During the implementation phase of the register, a computerized questionnaire was also designed and sent to all health administration centers in the country. A countrywide training program consisting of annual workshops at national, provincial, and district levels had been carried out for all staff who were working in the field.

Seven variables were selected for determination of injury patterns, ie, the specific location of occurrence of injury in the home environment $(n=10)$, injury mechanism $(n=16)$, type of injury $(\mathrm{n}=16)$, part of body injured $(\mathrm{n}=16)$, age and gender of the victim, and outcome $(n=4)$. A standardized registration form was used for data collection. Data were collected at hospital outpatient emergency departments, health care centers in rural and urban areas, and most peripheral health services in rural areas, known as "health houses". Data entered into a standard questionnaire was created in the Epi6 program, which was available to all health administration centers in the country. All centers were sending a disc to Iran's Center for Disease Control (CDC) in Tehran annually, and these were merged to form the national dataset. Quality control measures included checking for repeatability of cases, same-month revision of all completed forms, double data entry, missing data, and underreporting. This procedure was supposed to control $20 \%$ of all data registered in the first year and $10 \%$ in the following years. Supervisors of the program at the provincial and national levels monitored and controlled the data according to the plan. Monitoring excluded checking the completed forms and data entered in the computer system.

For this research, the national dataset was restricted to female injury victims for overall analysis and limited to those 15-49 years of age, generally known as "women of reproductive age". Data were analyzed using the Stata 11 statistical software package. Both descriptive and analytical methods were used. Frequency and relative frequency tables were produced. Age-adjusted incidence rates and mortality rates were plotted. The denominator for the five-year intervals of age needed to calculate incidence rates was estimated using averages of two national population censuses conducted in 1996 and 2006. The Chi-square test was used to assess predictors of mortality, followed by bivariate estimation of odds ratios along with their $95 \%$ confidence intervals. Finally, a logistic regression multivariate analysis was done while adhering to the standards of model development, as well as an assessment of model fitness and appropriateness. The analyses were done in accordance with the ethical codes of the Ministry of Health and Medical Education in Iran.

\section{Results}

Of the 307,064 home injuries reported during the years 2000-2002, about 152,600 cases (49.7\%) involved women. About half of these women $(76,474)$ belonged to the reproductive age group studied in this research. A histogram of the age distribution is given in Figure 1, and age-adjusted incidence rates are shown in Figure 2. The mean age of the women in the reproductive age group was $28 \pm 9.5$ years, with a median age of 26 years.

Overall, $56 \%$ of domestic injuries in the women of reproductive age occurred in the living room or a bedroom, $28.4 \%$ in the kitchen, $10.3 \%$ in the yard or garden, and the remainder occurred in other parts of the house, including the bathroom,

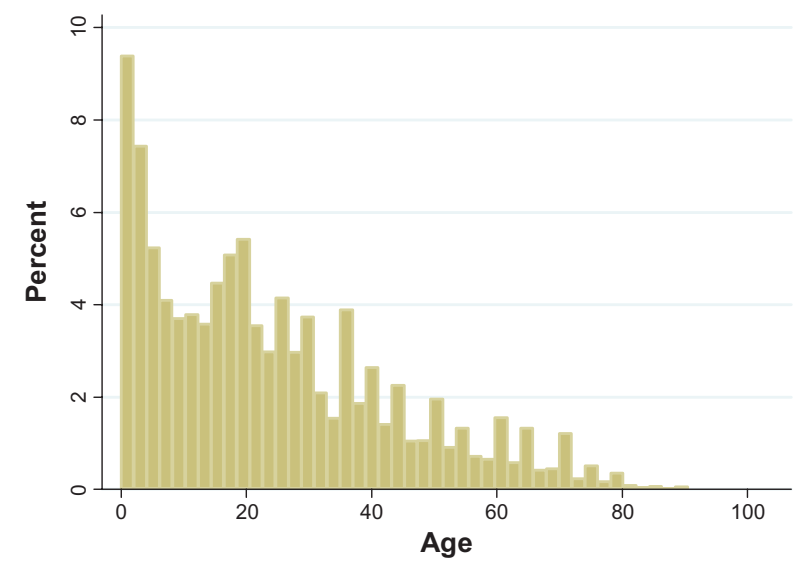

Figure I Histogram of age for Iranian women victims of injury. 


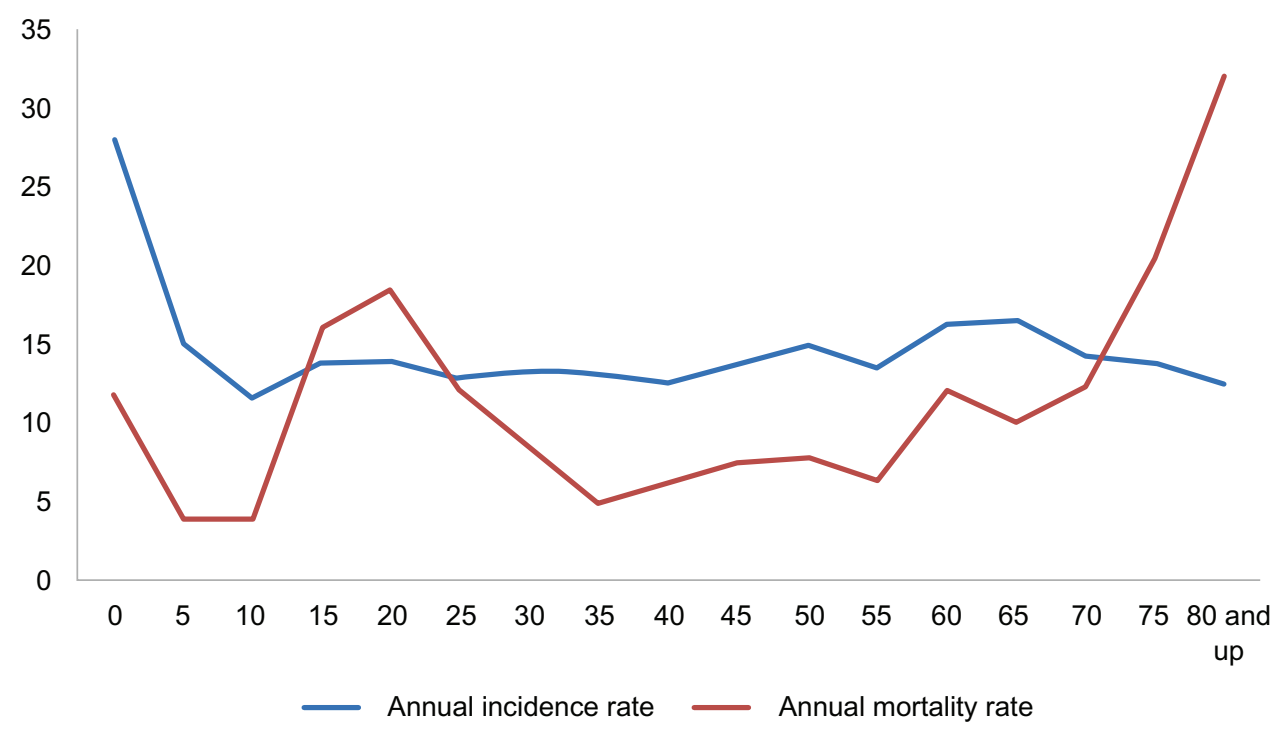

Figure 2 Age-adjusted incidence and mortality rates for Iranian female injury victims.

Notes: $y$ axis, age-adjusted rates as per 1000 for incidence and per 100,000 for mortality; $x$ axis, age groups of five-year intervals except for older than 80 years.

stable, stairs, or stockroom, or on the roof. Hands and fingers were injured in 37,934 cases (49.6\%), the lower limbs in 16,534 cases $(21.6 \%)$, with multiple trauma in 2104 cases $(2.8 \%)$, and involvement of other organs in the remaining cases. With regard to the mechanism of injury, $37.7 \%$ of all women and $32.3 \%$ of women in the reproductive age group were injured by hot liquids. The rest of the mechanisms of injury along with age group comparisons are shown in Table 1.

The majority (42.7\%) of injuries among women of reproductive age were burn wounds followed by lacerations
(32.6\%, Table 2). Age-adjusted incidence rates for female burn injury victims versus other types of injuries are compared in Figure 3 for the various age groups.

Eighty-five percent of suicides were the result of poisonings, followed by $11 \%$ due to burns. However, $45.2 \%$ of suicides by burning were fatal compared with a fatality rate of only $0.89 \%$ for poisoning. This means a 50 times higher risk of fatality for self-inflicted burns compared with poisoning.

For all the injuries, $42.5 \%, 27.2 \%$, and $30.3 \%$ were reported by primary health centers, secondary health centers,

Table I Distribution of injury mechanisms among the women injury victims in Iran compared for the three age groups

\begin{tabular}{|c|c|c|c|c|c|}
\hline Injury mechanism & $<\mathbf{I 5}$ years & Reproductive age & $>49$ years & Total & $P$ value \\
\hline Attack and sting & 691 & 745 & 271 & 1707 & $<0.001$ \\
\hline Collision with sharp objects & 8931 & 22,520 & 4,437 & 35,888 & $<0.001$ \\
\hline Collision with substance & 3181 & 3821 & 1,212 & 8214 & $<0.00$ I \\
\hline Contact with hot objects & 3934 & 4033 & 1,233 & 9200 & $<0.001$ \\
\hline Exposure to fire & 1782 & 3206 & 781 & 5769 & $<0.001$ \\
\hline Hot liquids & 26,328 & 24,699 & 6501 & 57,528 & $<0.001$ \\
\hline Falls & 4388 & 3927 & 1936 & $|0,25|$ & $<0.001$ \\
\hline Overthrown (fall at the same level) & 4408 & 4316 & 2190 & 10,914 & $<0.001$ \\
\hline Foreign body & 493 & 661 & 115 & 1269 & $<0.01$ \\
\hline Chemical materials & 218 & 546 & 60 & 824 & $<0.001$ \\
\hline Violence & 130 & 539 & 73 & 742 & $<0.001$ \\
\hline Others & 376 & 638 & 147 & $116 \mid$ & $<0.01$ \\
\hline Suicide & 142 & 2,906 & 106 & 3154 & $<0.001$ \\
\hline Overcome by smoke & 94 & 365 & 58 & 517 & $<0.001$ \\
\hline Using drugs or poison & 1424 & 3283 & 147 & 4854 & $<0.001$ \\
\hline Falling debris & 115 & $|5|$ & 65 & 331 & $<0.001$ \\
\hline Drowning & 38 & 6 & 3 & 47 & $<0.001$ \\
\hline Electric shock & 91 & 112 & 27 & 230 & $<0.001$ \\
\hline Total & 56,764 & 76,474 & 19,362 & 152,600 & $<0.001$ \\
\hline
\end{tabular}


Table 2 Types of injuries among women trauma victims in Iran

\begin{tabular}{lllll}
\hline Kind of injury & Under I5 years & Reproductive age & Above 49 years & Total \\
\hline Amputation & 116 & 134 & 53 & 303 \\
Bleeding & 152 & 218 & 56 & 426 \\
Brain trauma & 342 & 217 & 91 & 650 \\
Bumping & 1604 & 2669 & 921 & 5194 \\
Burn & 32,182 & 32,620 & 8593 & 73,395 \\
Contusion & 1047 & 1165 & 454 & 2666 \\
Cuts and lacerations & 11,909 & 24,963 & 5085 & 41,957 \\
Eye injury & 189 & 267 & 22 & 478 \\
Fractures & 3189 & 3174 & 2265 & 8628 \\
Multiple & 52 & 87 & 32 & 171 \\
Others & 199 & 300 & 52 & 551 \\
Pain & 280 & 413 & 119 & 812 \\
Poisoning & 1755 & 6280 & 295 & 8330 \\
Shock & 67 & 69 & 18 & 154 \\
Suffocation & 147 & 164 & 34 & 345 \\
Crush injuries & 3534 & 3734 & 1272 & 8540 \\
Total & 56,764 & 76,474 & 19,362 & 152,600 \\
\hline
\end{tabular}

and hospitals, respectively. Of the female cases, 1029 died, 174 became disabled, and the remainder improved or were undergoing therapy when reported. Multivariate analysis found that age group, injury mechanism, and injured body part were significant predictors of death. On bivariate analysis, women of reproductive age had the highest likelihood of death compared with those who were younger or older. The adjusted odds ratio of death, through multivariate analysis, for the reproductive age group remained higher than for childhood injuries, but was slightly lower than the figure for the group aged 50 years and older. The adjusted odds ratios from the multivariate analysis are given in Table 3.
Among women of reproductive age, the risk of dying after injury was 5.5 times higher for burns versus other types of injury (95\% confidence interval 4.6-6.6).

\section{Discussion}

In this study, the incidence of injuries were found to be higher among preschool-aged children. This is in line with previous overall and injury-specific studies. ${ }^{6,7}$ Younger middle-aged women, although they had lower incidence rates, had higher mortality rates, indicative of higher case fatality in the early reproductive years. Consistent results from multivariate analysis indicated that women of reproductive age had a

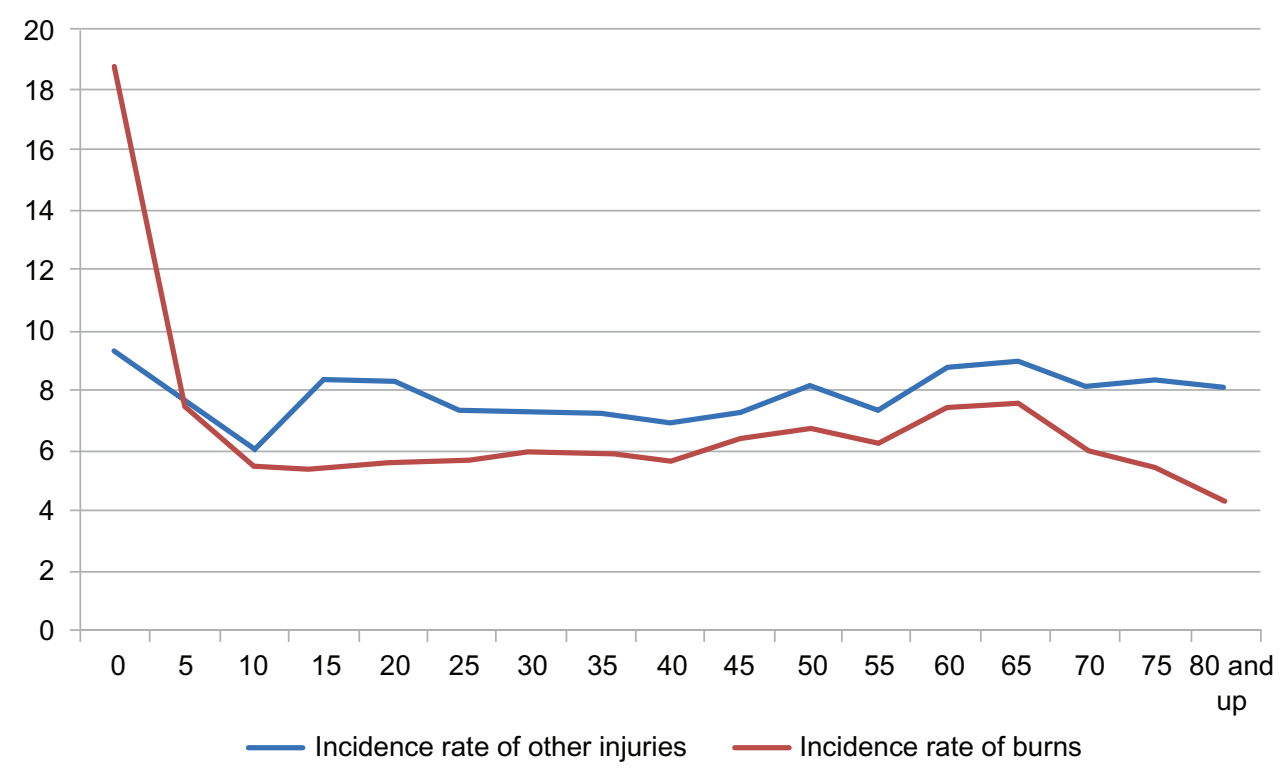

Figure 3 Age-adjusted incidence rates for Iranian female burn injury victims versus other types of injuries. Notes: $x$ axis, age groups of five-year intervals except for over 80 years; $y$ axis, age-adjusted rates as per 1000. 
Table 3 Adjusted odds ratios for injury death predictors derived from logistic regression analysis of Iranian national registry data

\begin{tabular}{llll}
\hline Death predictors & \multicolumn{3}{l}{ OR [95\% Cl] } \\
\cline { 2 - 4 } & Reference group & \\
\hline Injury mechanism & & & \\
Attack and sting & & & \\
Collision with sharp objects & 0.1 & 0.0390539 & 0.3863006 \\
Collision with substance & 0.5 & 0.1582152 & 1.535345 \\
Contact with hot objects & 1.9 & 0.7130849 & 5.57533 \\
Exposure to fire & 27.6 & 10.28564 & 74.12296 \\
Hot liquids & 0.8 & 0.2783321 & 2.064572 \\
Falls & 1.8 & 0.6655159 & 5.115443 \\
Overthrown (fall at the same level) & 0.5 & 0.1512783 & 1.404848 \\
Foreign body & 2.8 & 0.8299897 & 9.202418 \\
Chemical materials & 30.4 & 10.95673 & 84.18282 \\
Violence & 6.1 & 2.008061 & 18.58508 \\
Others & 7.4 & 2.533444 & 21.49362 \\
Suicide & 31.8 & 11.78978 & 85.76353 \\
Overcome by smoke & 28.6 & 10.08895 & 80.84251 \\
Using drugs or poisons & 3 & 1.074721 & 8.577741 \\
Falling debris & 9.8 & 3.072298 & 31.24254 \\
Drowning & 222.9 & 69.42403 & 716.0821 \\
Electric shock & 21.7 & 6.829051 & 68.69778 \\
Age group & & & \\
< I5 years & & & \\
Reproductive age & 1.7 & 1.409415 & 1.986962 \\
Beyond reproductive age & 2.1 & 1.689494 & 2.628329 \\
Body part & & & \\
Head and neck injuries & 2.6 & 1.980075 & 3.407706 \\
and brain trauma & & & \\
\hline
\end{tabular}

higher likelihood of death compared with younger women. Although this group of injury victims are not well addressed in the research literature, some burn studies have reported such a pattern. ${ }^{8}$

The mortality slope was found to start increasing after the age of 10 years, and remaining high for up to the first two decades of life. In Iranian culture, women are responsible for doing household chores, mainly cooking and cleaning. Some young females are employed in households that are not their own, while others are obliged to work in their home as family members. ${ }^{9}$ The risk may decrease in later life for two reasons; the first is that women become more skilled as they get older, and the second is that, through the later phase of their reproductive life, their daughters grow older and share both the responsibilities and the risks.

The majority of injuries among women of reproductive age were burns, which are among the leading causes of death and disability worldwide. ${ }^{10}$ Traffic accidents rank first in injury morbidity and mortality in many countries, including Iran. Burns rank second in childhood and third overall among injuries in Iran. ${ }^{11,12}$ Although little research has focused on injuries in the reproductive age group, some regional studies have found burns to be catastrophic injuries during pregnancy. ${ }^{13-15}$ Nevertheless, when it comes to domestic injuries among women of reproductive age, our research showed that burns are the leading cause of both morbidity and mortality. This could be due to the fact that cooking at home is the most important domestic responsibility of women in Iran. Burns, cuts, and lacerations may be the most common risks of unsafe cooking practices. A major concern in this regard would be safety procedures for cooking at home. Cooking safety is dependent on the safety of cooking appliances, and unsafe behaviors and a dangerous cooking environment all need to be addressed in prevention work. ${ }^{16-18}$ Although women of reproductive age are at higher risk of cookingrelated injuries, cooking is not the sole source of domestic injuries in women and, like other family members, they are also susceptible to other sources of injury.

As could be inferred from Table 1, women of reproductive age are more likely to be involved in violence and injuries related to suicide. We found that self-inflicted burns were 50 times more likely to be fatal compared with suicide by poisoning. Self-inflicted burns are shown to be a major public health problem in Iran, and particularly so among young women. ${ }^{19,20}$ The attention of health policy-makers in Iran needs to be drawn to this problem. Regardless of regional variations in suicide patterns, mortality from suicide is undoubtedly a major public health problem. According to a World Health Organization report, approximately one million people worldwide died as a result of suicide in $2000 .^{21}$

A large number of staff are currently employed in the Iranian health care system to work specifically in the field of reproductive health and primary health care in childhood, so it seems quite reasonable to consider possible integration of a focused national injury prevention and safety promotion program into the current health service package delivered to women of reproductive age.

Like any other register-based study, we were able to study only the most important variables of interest in injury research because of the minimum data requirement in national registers. Underregistration of death counts is common in death registry systems, and it is even higher in event-disease registers that also collect information about outcome measures, including mortality. ${ }^{22}$ Our study is not an exception, and the injury incidence and mortality rates presented here should be interpreted as minimum rates due to inevitable underestimation.

\section{Disclosure}

The authors report no conflicts of interest in this work. 


\section{References}

1. Krug EG, Sharma GK, Lozano R. The global burden of injuries. Am J Public Health. 2000;90(4):523-526.

2. Sadeghi-Bazargani H. Injury epidemiology and publishing injury research. J Inj Violence Res. 2012;4(1):1.

3. Arshi S, Sadeghi-Bazargani H, Mohammadi R, Mohammad Zadeh MS, Rouhi A, Barak M. Burns comprising 3/4th of home injuries in preschool children of rural areas of Ardabil province, Iran. J Med Sci. 2007; $7(2): 248-251$.

4. Sadeghi-Bazargani H, Mohammadi R, Svanstrom L, et al. Epidemiology of minor and moderate burns in rural Ardabil, Iran. Burns. 2010;36(6):933-937.

5. Mohammadi R, Ekman R, Svanstrom L, Gooya MM. Rationales for home safety promotion in the Iranian primary healthcare system: results from a pilot study. Public Health. 2006;120(1):58-64.

6. Allorto NL, Oosthuizen GV, Clarke DL, Muckart DJ. The spectrum and outcome of burns at a regional hospital in South Africa. Burns. 2009;35(7):1004-1008.

7. MacKenzie EJ. Epidemiology of injuries: current trends and future challenges. Epidemiol Rev. 2000;22(1):112-119.

8. Sadeghi-Bazargani H, Mohammadi R. Epidemiology of burns in Iran during the last decade (2000-2010): review of literature and methodological considerations. Burns. 2011;38(3):319-329.

9. Mohammadi R, Sadeghi-Bazargani H, Gooya MM. Burns to young working females in work and home settings. Box 4.2 in: World Report on Child Injury Prevention. World Health Organization: Geneva, Switzerland; 2008.

10. Murray CJ, LopezAD. Global mortality, disability, and the contribution of risk factors: Global Burden of Disease Study. Lancet. 1997; 349(9063): 1436-1442.

11. Naghavi M, Djafari N, Alaeddini F, Akbari I. Injury Epidemiology in the Islamic Republic of Iran. 1st ed. Tehran, Iran: Iranian Ministry of Health Publications; 2004.
12. Naghavi M, Abolhassani F, Pourmalek F, Jafari N, Moradi M, Eshrati B. Burden of diseases and injuries in Iran. Iran J Epidemiol. 2009;4(1):1-19.

13. Pacheco LD, Gei AF, VanHook JW, Saade GR, Hankins GD. Burns in pregnancy. Obstet Gynecol. 2005;106(5 Pt 2):1210-1212.

14. Mabrouk AR, el-Feky AE. Burns during pregnancy: a gloomy outcome. Burns. 1997;23(7-8):596-600.

15. Maghsoudi H, Samnia R, Garadaghi A, Kianvar H. Burns in pregnancy. Burns. 2006;32(2):246-250.

16. Peck MD, Kruger GE, van der Merwe AE, Godakumbura W, Ahuja RB. Burns and fires from non-electric domestic appliances in low and middle income countries. Part I: the scope of the problem. Burns. 2008; 34(3):303-311.

17. Peck MD, Kruger GE, van der Merwe AE, et al. Burns and injuries from non-electric-appliance fires in low- and middle-income countries Part II: a strategy for intervention using the Haddon matrix. Burns. 2008; 34(3):312-319.

18. Sadeghi BH, Arshi S, Ekman R, Mohammadi R. Prevention-oriented epidemiology of burns in Ardabil provincial burn centre, Iran. Burns. 2011;37(3):521-527.

19. Maghsoudi H, Garadagi A, Jafary GA, et al. Women victims of self-inflicted burns in Tabriz, Iran. Burns. 2004;30(3):217-220.

20. Ahmadi A, Mohammadi R, Stavrinos D, Almasi A, Schwebel DC. Self-immolation in Iran. J Burn Care Res. 2008;29(3):451-460.

21. De Leo D, Bertolote J, Lester D. World Report on Violence and Health. World Health Organization; Geneva, Switzerland; 2002.

22. Mathers C, Lopez A, Salomon J, Ezzati M. National burden of disease studies: a practical guide. World Health Organization: Geneva, Switzerland; 2001.
International Journal of General Medicine

\section{Publish your work in this journal}

The International Journal of General Medicine is an international, peer-reviewed open-access journal that focuses on general and internal medicine, pathogenesis, epidemiology, diagnosis, monitoring and treatment protocols. The journal is characterized by the rapid reporting of reviews, original research and clinical studies across all disease areas.

\section{Dovepress}

A key focus is the elucidation of disease processes and management protocols resulting in improved outcomes for the patient.The manuscript management system is completely online and includes a very quick and fair peer-review system. Visit http://www.dovepress.com/ testimonials.php to read real quotes from published authors. 